\title{
THE EXTREME POSITION AND AMPLITUDE AMPLIFICATION OF BICHROMATIC WAVES PROPAGATION BASED ON THIRD ORDER SOLUTION OF BOUSSINESQ EQUATION
}

\author{
MARWAN AND ANDONOWATI
}

\begin{abstract}
This paper concerns with the down-stream propagation of waves over initially still water. Such a study is relevant to generate waves of large amplitude in wave tank of a hydrodynamic laboratory. Input in the form of a time signal is provided at the wave-maker located at one side of the wave tank; the resulting wave then propagates over initially still water towards the beach at the other side of the tank. Experiments show that nonlinear effects will deform the waves and may lead to large wave with waves height larger than twice the original input; the deformation may show itself as peaking and splitting. It is direct scientific interest to understand and quantify the nonlinear distortion; it is also much practical interest to know at which location in the wave tank, the extreme position, the waves will achieve their maximum amplitude and to know the amplitude amplification factor. To investigate this, a previously introduced concept called Maximal Temporal Amplitude (MTA) is used: at each location the maximum over time of the wave elevation. An explicit expression of the MTA cannot be found in general from the governing equations and generating signals. In this paper we will use Boussinesq model and third order approximation theory to calculate the approximate extreme positions and amplification factor for wave-group that originate from initially bi-chromatic type of wave. The wave is described by superposition of two monochromatic waves. We show that, the extreme position depends on the amplitude and the wave length of the group. The theoretical results are verified with numerical as well as experimental results for comparison.
\end{abstract}

Received 11-04-2008, Accepted 27-11-2008.

2000 Mathematics Subject Classification: 35Q35, 35Q53

Key words and Phrases: Boussinesq equation, MTA, wave propagation, asymtotic expansion, AAF, extreme position.

Marwan: Department of Mathematics, Faculty of Mathematics and Natural Sciences, Universitas Syiah Kuala, Jl. Tgk. Daud Bereuh No 4, Darussalam-Banda Aceh 23111, Indonesia.

E-mail: marwan.ramli@math-usk.org

Andonowati: Study Programm of Mathematics, Faculty of Mathematics and Natural Sciences, Institut Teknologi Bandung, Bandung, Indonesia. 